\section{Las reformas de salud neoliberales en América Latina: una visión crítica a través de dos estudios de caso}

\author{
Nuria Homedes ${ }^{1}$ \\ y Antonio Ugalde 2
}

Palabras clave: reforma del sector salud, descentralización, privatización, América Latina, Colombia, México.

\footnotetext{
Escuela de Salud Pública, Houston Health Science Center, Universidad de Texas, Estados Unidos de América. La correspondencia debe dirigirse a Nuria Homedes, 1100 N. Stanton, Suite 110, El Paso, TX 79902, Estados Unidos de América. Correo electrónico: nhomedes@utep.edu

2 Departamento de Sociología, Universidad de Texas-Austin, Estados Unidos.
}

Desde la década de los años setenta había conciencia de que los sistemas de salud latinoamericanos eran poco equitativos e ineficientes y de que los niveles de salud de América Latina estaban por debajo de lo que podía esperarse debido a su bajo desarrollo económico. A partir de la Declaración de Alma Ata de 1978, en la que se adoptó la meta de salud para todos (1), los ministerios de salud de países de la Región empezaron a extender los servicios de salud a poblaciones marginadas y rurales, siguiendo los principios allí enunciados. El modelo aprobado en Alma Ata está basado en principios de solidaridad y universalidad en el acceso a los servicios de salud, de eficacia en función del costo (mediante la promoción de los servicios de atención primaria y de prevención) y en la participación comunitaria (lo que más tarde se ha denominado democratización de los servicios de salud). Sin embargo, tanto la orientación biomédica de la atención sanitaria como la crisis económica que empezó poco tiempo después abortó la incipiente iniciativa.

Para superar la crisis económica que comenzó alrededor de 1980, el Fondo Monetario Internacional (FMI) exigió a los gobiernos que recortaran los gastos en los sectores sociales, entre ellos el sector salud (los llamados ajustes estructurales). La reducción de los presupuestos de salud provocó un deterioro aun mayor de los servicios y el aumento de la insatisfacción de los proveedores y de los usuarios. El Banco Mundial aprovechó la crisis del sector para fortalecer su programa de préstamos al sector de la salud y a finales de la década de 1990 se había convertido en la agencia internacional que más fondos prestaba a los países en vías de desarrollo para ese sector. En 1999, según los precios de 1996, el Banco Mundial había concedido préstamos por un valor total de US\$ 16800 millones (2).

Apoyado en el poder de sus recursos, el Banco Mundial ha promovido una reforma de salud basada en los principios básicos de que el sector privado es más eficiente que el sector público y de que la función del Gobierno es regular y no proveer servicios. Siguiendo su ideología neoliberal, el Banco Mundial propone la privatización de los servicios, su descentralización, la separación de las funciones de financiación y provisión, y la universalización del acceso a un paquete de servicios mínimos que cada país debe definir de acuerdo con sus recursos y estudios de eficiencia en función de los costos.

En las políticas promovidas por el Banco Mundial, ninguna intervención ha contribuido a 
mejorar apreciablemente los niveles de salud de la población. Un elemento al que se ha dado muy poca importancia en esas intervenciones es el de los recursos humanos. En otro artículo publicado en este número de la Revista Panamericana de Salud Pública se señala la necesidad de darles a los recursos humanos participación en las reformas y se exploran las posibles razones por las cuales quedaron excluidos de ellas (3). Un análisis semejante se podría hacer acerca de las políticas farmacéuticas y de los programas de salud pública, tales como la promoción de la salud, la salud ocupacional y el control del medio ambiente, ninguno de los cuales ha formado parte de las reformas liberales.

En el presente artículo se analizan las experiencias de América Latina relacionadas con la descentralización y la privatización de los servicios de salud. Se presentan dos estudios de caso: Colombia, 11 años después de haber aprobado la Ley 100 de 1993, y México, a los 20 años de haber iniciado la descentralización de la Secretaría de Salud.

\section{LA PRIVATIZACIÓN EN AMÉRICA LATINA}

La mayoría de los países de América Latina se han opuesto a la privatización de la prestación de servicios de salud financiados por el sector público o por sistemas de seguridad social. Brasil tiene una larga historia de contratación de servicios hospitalarios al sector privado sin fines de lucro. Otros países, como Costa Rica, El Salvador y más recientemente México, han experimentado con planes piloto de contratación pública de servicios privados (4).

Chile, que había tenido un sistema nacional de salud con cobertura universal y de acceso gratuito entre 1952 y 1981, fue el primer país latinoamericano en aplicar una reforma neoliberal a principios de la década de 1980. La dictadura fragmentó el sistema nacional de salud y creó aseguradoras privadas denominadas Institutos de Salud Provisional (ISAPRE). También descentralizó la atención primaria y hospitalaria mediante su transferencia a las municipalidades y a las áreas de salud, respectivamente, y en 1986 estableció cuotas de recuperación en el sector público. Según evaluaciones realizadas, la reforma aplicada en Chile:

a) segmentó el sistema, lo que provocó una mayor inequidad en el acceso a los servicios, ya que solo los ricos tienen los recursos económicos para optar por los servicios de los ISAPRE. La segmentación no mejoró la eficiencia del sistema; el gasto per cápita de los beneficiarios de los ISAPRE es aproximadamente el doble del gasto per cápita en el sector público (22\% de la población consume $46 \%$ del gasto para la salud), a pesar de que los beneficiarios de los ISAPRE son, en promedio, más jóvenes y sanos

b) benefició a los ISAPRE, que llegaron a funcionar con márgenes de beneficio de $20 \%$ y gastos administrativos de otro $20 \%$, debido a prácticas que la superintendencia de ISAPRE no ha podido controlar, como la selección cuidadosa de sus afiliados (jóvenes y sanos) y los subsidios directos del Estado (alrededor de 4\% del gasto de los ISAPRE).

c) aumentó la inequidad entre las áreas rurales y urbanas

d) redujo el presupuesto público del sector de la salud, que además tiene la difícil tarea de acoger a las personas que en períodos de crisis económica dejan de contribuir a ISAPRE y pasan a depender del sector público.

La reforma de salud de Colombia, aplicada en 1993, es la que siguió más de cerca las recomendaciones del Banco Mundial (5), a la vez que intentó corregir algunos de los problemas identificados durante el proceso de la reforma chilena.

\section{LA REFORMA COLOMBIANA}

La Ley 100 de 1993 reformó el sistema de salud y apostó por el modelo de competencia regulada o pluralismo estructurado (6). Para evitar generar la inequidad producida por la reforma chilena, en Colombia se crearon un fondo de solidaridad y un plan obligatorio de servicios. El nuevo sistema se basa en el aseguramiento de toda la población mediante el Sistema General de Seguridad Social en Salud (SGSSS) con dos regímenes: el contributivo y el subsidiado. El primero, que cubre a $29 \%$ de la población, es para los trabajadores y pensionados con ingresos por encima de un nivel establecido por el Sistema de Identificación y Clasificación de Potenciales Beneficiarios para los Programas Sociales y que contribuyen con $12 \%$ de sus ingresos al Fondo de Solidaridad y Garantía. Este fondo paga una cantidad fija por persona asegurada - denominada unidad de pago por capitación (UPC) - a la administradora de salud con la que elija afiliarse el contribuyente. Las UPC de los beneficiarios del régimen subsidiado ( $26 \%$ de la población) se cubren con los recursos del Fondo de Solidaridad y Garantía. El Fondo de Solidaridad y Garantía también se utiliza para subsidiar el pago de las UPC de los contribuyentes que cobran menos de tres salarios mínimos. Mientras no se logre el aseguramiento de la población en su totalidad, habrá otra categoría de pacientes, los vinculados (45\% de la población), constituida por las personas que no están afiliadas a ninguno de los regímenes anteriores y que utilizan los servicios públicos. 
La administración de los servicios se hace por medio de entidades promotoras de la salud (EPS) o administradoras del régimen subsidiado (ARS). Las EPS son empresas que pueden ser privadas o públicas y que administran servicios y contratan a los proveedores, tanto para el plan contributivo como para el subsidiado. La prestación de servicios se hace mediante redes de instituciones prestadoras de salud, ya sean públicas o privadas, que tienen la obligación de proveer un plan de prestaciones - denominado plan obligatorio de salud (POS) - a los beneficiarios del régimen contributivo (POS-C) y otro a los del régimen subsidiado (POS-S). La ley preveía que a medida que aumentase el financiamiento para los servicios de salud se ampliaría el POS-S y, finalmente, todos los colombianos tendrían acceso al mismo plan de servicios, independientemente de su capacidad de pago. Sin embargo, la crisis económica ha impedido que esto se logre y en estos momentos, la UPC del POS-C es casi el doble de la UPC del POS-S (US\$ 126,00 frente a US\$ 68,00, respectivamente) (7), y este último no abarca a la mayoría de los servicios de salud de los segundo y tercer niveles de atención.

Adicionalmente, todos los ciudadanos que lo deseen pueden comprar un seguro complementario que les permite acceder a servicios no abarcados por el POS. Con la reforma, el número de familias con seguro complementario se ha reducido en $30 \%$ y en la actualidad solo 700000 colombianos están afiliados a un seguro complementario.

En el área de la salud pública, la Ley 100 obliga a los municipios y departamentos a poner en marcha el Plan de Atención Básica. Este plan — que debe ser parte integral de los planes local y departamental de salud y de los planes de desarrollo de las entidades territoriales correspondientesdebe, esencialmente, articular las acciones de todos los sectores y fuerzas vivas de la sociedad en aras de formular y desarrollar políticas saludables, crear ambientes favorables a la salud en las dimensiones físicas y sociales, desarrollar aptitudes personales y colectivas para proteger y fomentar la salud y redirigir los servicios de salud hacia la prevención de las enfermedades y la protección de la salud (8).

\section{Impacto de la reforma}

Para noviembre de 2004 se había generalizado en el país una gran preocupación por el estado del sistema de salud, a tal punto que en el Congreso se habían recibido 14 proyectos de ley para "reformar" la reforma. Los defensores de la reforma proponían solucionar los problemas mediante el mejoramiento de la gestión y la regulación, pero otros apostaban por cambios más radicales. La situación se puede resumir como sigue:

La salud pública. La falta de una política y de reglamentos claros, la desarticulación de los equipos entrenados y el desinterés del Gobierno hicieron que se descuidaran mucho las actividades de salud pública. Por ejemplo, la cobertura de vacunación con la tercera dosis de la vacuna antipoliomielítica que debe aplicarse antes del primer año de vida descendió de 94\% en 1995 a 80\% en 2003 (9). Según las encuestas de demografía y salud de 1990, 1995 y 2000, el porcentaje de niños entre 12 y 23 meses de edad que recibieron todas las vacunas disminuyó de $83 \%$ en 1990 a $71 \%$ en 1995 y a 66\% en 2000 (10). La incidencia de malaria aumentó desde la década de 1960, pero su incremento se acentuó a partir de 1998, mientras que la incidencia de dengue clásico ha registrado un promedio anual de 30332 casos en el quinquenio de 1996-2001.

Por su parte, el control de la tuberculosis ha perdido calidad, en parte porque el programa se ha fragmentado: las instituciones prestadoras de atención de salud son las responsables de atender al paciente y a los contactos que están afiliados al sistema, mientras que los municipios, mediante el plan de atención básica, son los encargados de entregarles el tratamiento a los pacientes sin seguro y de hacer el seguimiento de sus contactos. Las instituciones prestadoras de servicios de salud indican que las presiones de tiempo les impiden hacer una buena anamnesia y estudiar a los contactos. El resultado final es que en tres municipios que estudiaron Ayala Cerna y Kroeger, el número de pacientes en tratamiento se había reducido y no se hacía el seguimiento de los contactos (11).

\section{Afiliación y equidad del acceso a los servicios}

Aunque el número de personas aseguradas ha aumentado, el proceso de afiliación se ha estancado y en la actualidad, más de 10 años después de iniciada la reforma, $45 \%$ de los colombianos no disponen de seguro de salud. Esto se explica, en parte, por la crisis económica - en la última década el empleo permanente se ha reducido en $30 \%$ y el empleo temporal se ha duplicado- y por las dificultades del sistema para captar a la población "informal". Según el gobierno municipal de Santa Fe de Bogotá, es necesario cambiar el sistema de financiamiento para alcanzar la cobertura universal, pues cerca de $30 \%$ de la población no es lo suficientemente pobre para recibir el subsidio del Estado, pero tampoco cuenta con los ingresos suficientes para mantener su afiliación al régimen contributivo (12). Se debe aclarar que la afiliación al seguro no garantiza el acceso. 
El Grupo de Economía de la Salud (13) analizó los datos de una encuesta realizada en 1997 sobre la calidad de vida y encontró que entre los encuestados que habían padecido alguna enfermedad en los 30 días previos a la encuesta, la posibilidad de que acudieran a los servicios de salud dependía del tipo de seguro que los cubría. Así, mientras solo $14 \%$ de los afiliados al régimen contributivo dejaron de recibir servicios de salud, $31 \%$ de los afiliados al régimen subsidiado y $40 \%$ de los que no estaban afiliados a ninguno de los regímenes anteriores (vinculados) no recibieron servicios. Además, mientras que en el régimen contributivo la principal razón para no acudir al servicio médico fue que la enfermedad era leve $(45,4 \%)$, para los subsidiados y vinculados el motivo principal fue la falta de dinero ( $43,9 \%$ y $63,4 \%$, respectivamente). Otros autores han señalado que la adopción del seguro para los pobres no se ha traducido en un aumento importante de la demanda $(14,15)$ y que las cuotas moderadoras y los pagos compartidos podrían estar restringiendo el acceso más allá de los esperado (16). Los gastos de bolsillo parecen haber disminuido en todos los estratos sociales, pero más aun en las personas de nivel socioeconómico alto (17). Flórez y Tono (18) demostraron que la inequidad entre las áreas rurales y urbanas se mantiene, tanto en el aseguramiento como en la utilización de servicios y en los niveles de salud.

Entrevistas realizadas en noviembre de 2004 a médicos y estudiosos y a usuarios del sistema de salud revelaron la existencia de barreras geográficas y administrativas que impiden el acceso a los servicios. Por ejemplo, $78 \%$ de los proveedores de servicios de salud en Santa Fe de Bogotá se ubican en el norte de la ciudad, donde residen los usuarios con ingresos más altos (12). Por otra parte, se observa un retraso de más de un año en el pago a los prestadores de servicios - que debería realizarse en los 60 días posteriores al rendimiento del servicio-, especialmente en pacientes del régimen subsidiado, lo que repercute negativamente en los prestadores, quienes acaban negando sus servicios a los afiliados que residen en las municipalidades "morosas" (9).

Limitada capacidad de elección y monopolios. Al igual que en los Estados Unidos de América, el número de EPS y, en especial, de ARS, se ha reducido (7) y cuatro EPS controlan más de 50\% de los ingresos totales del sector. Restrepo y colaboradores (19) encontraron que el mercado de servicios de salud era de carácter monopólico en $65 \%$ de los municipios, que en $30 \%$ de ellos era oligopólico y que solo en $5 \%$ de los municipios era competitivo.

Una encuesta de la Defensoría del Pueblo reveló que la posibilidad de escoger al prestador de servicios disminuyía a medida que los usuarios re- querían servicios más especializados. De los encuestados, 39\% manifestaron que no habían podido elegir a su médico general, $62 \%$ no habían tenido la posibilidad de escoger al especialista, $63 \%$ no había podido elegir en qué hospital someterse a cirugía y $68 \%$ no había podido elegir dónde hacerse una prueba diagnóstica. Las restricciones a la libertad de elección son mayores en el régimen subsidiado que en el contributivo (20).

Elevados gastos administrativos y de promoción. En 1997, 26\% de los gastos de las EPS se destinaban para fines administrativos (incluidos los salarios), $17 \%$ eran para promoción y solo $57 \%$ eran para servicios. Estos porcentajes mejoraron durante 2001: $77 \%$ se dedicaron a los servicios, $18 \%$ a gastos administrativos y solo $4,5 \%$ a actividades de promoción (7). Posiblemente, a medida que se consolidaban los monopolios, los gastos de promoción descendieron. Profesionales sanitarios y estudiosos del sistema de salud colombiano revelaron en noviembre de 2004 que los beneficiarios de la reforma son las EPS y las ARS, ya que - al igual que las aseguradoras estadounidenses- siempre negocian con las empresas proveedoras de servicios contratos que les generan más beneficios, aunque estas se vean obligadas a sacrificar la calidad de los servicios que prestan. Además, al contrario de lo programado, muchas administradoras tienen su propia red de instituciones prestadoras de servicios, es decir, que hay una integración vertical que les permite reducir aun más los gastos. Esto menoscaba los ingresos de los prestadores de servicios y disminuye la competitividad de las prestadoras públicas, porque los salarios de sus trabajadores, por ser empleados de la administración pública, no se pueden reducir tan fácilmente como los de los trabajadores de las empresas privadas.

Debilidad de las autoridades reguladoras y corrupción. La regulación la ejercen tres organismos: el Ministerio de Protección Social, que define las políticas y emite normas; la Superintendencia Nacional de Salud, que está encargada de inspeccionar, vigilar y controlar la conducta de los agentes del sistema; y el Consejo Nacional de Salud, que se encarga de definir el contenido y de valorar los planes, el monto de las cotizaciones y la distribución de los recursos. Lamentablemente, estos organismos no pueden cumplir con las funciones asignadas y se han documentado problemas importantes en el control del sistema. Por ejemplo, se calcula que la evasión de aportes al Fondo de Solidaridad y Garantía es de $36 \%$ a $49 \%$ de lo recaudado (7) y estos fondos - que tienen un superávit importante- no se han revertido en el fortalecimiento del sistema, sino que se han invertido en títulos y bonos que fi- 
nancian otros gastos del Gobierno central (9). La regulación estatal en el régimen contributivo ha sido muy errática y casi inexistente. Las EPS privadas aceptan a los pacientes más sanos y refieren a los que necesitan servicios más caros a los administradores públicos (21). En 2001, las EPS privadas cobraban la UPC de 50000 fallecidos (7).

También se han observado altos niveles de corrupción en las administradoras del régimen subsidiado y en las administraciones municipales y departamentales. Entre las manifestaciones de corrupción se encuentran los pagos de las ARS a los alcaldes para trasladar usuarios a su favor; cobros de UPC por afiliados inexistentes y carnés no entregados, pagos de prestadoras de servicios a las ARS para obtener contratos; incorporación al régimen subsidiado de personas con capacidad de pago; $\mathrm{co}^{-}$ bros por vacunas y otros servicios gratuitos, y retrasos en los pagos a las ARS.

En diciembre de 2003, la deuda de las administraciones municipales a las ARS era equivalente a más de US\$ 155 millones (9). Esto explica en gran parte el colapso de la red hospitalaria contratada para la prestación del POS-S. La falta de fondos en los hospitales públicos hace que falten insumos y que se deteriore la calidad de los servicios. Cuando hay problemas, el Estado termina pagando el valor del rescate o la liquidación de los aseguradores y servidores públicos (9).

La calidad de la atención. No hay estudios que permitan determinar si la calidad de los servicios ha mejorado; sin embargo, es posible afirmar que todavía queda mucho por mejorar. Un estudio sobre la práctica de cesáreas en cuatro hospitales de Cali reveló que 81,2\% de las cesáreas eran injustificadas y que la cifra era mayor en los hospitales privados que en los públicos (22). En una encuesta realizada a 417 pacientes diabéticos seleccionados mediante muestreo sistemático simple se encontró que 56,5\% de ellos no recibieron los medicamentos (hipoglucemiantes e insulinas) que forman parte del POS y tuvieron que comprarlos; $34 \%$ de estos pacientes pertenecían al régimen contributivo y $22,5 \%$ al subsidiado. En cambio, del $43,5 \%$ de los pacientes que recibieron los medicamentos sin costo alguno, 90,5\% pertenecían al régimen contributivo, 7,5\% al subsidiado y $2 \%$ al vinculado (23). McPake y colaboradores (24) no encontraron diferencias en cuanto a la calidad de la atención brindada en 1997 y 1998, pero los comentarios de los proveedores de salud fueron preocupantes: "Estamos perdiendo la calidad humana", "Ahora todo es cobrar, cobrar", "La calidad debería ser nuestro objetivo, pero no lo es porque tenemos que convertir los números en pesos". Según estos entrevistados, la mayoría de los cambios se habían producido en el área administra- tiva y se había descuidado la capacitación del personal clínico. Como se señala en otro artículo de este número especial, $62 \%$ de los médicos especialistas colombianos que participaron en una encuesta dijeron que la calidad del sistema se había deteriorado y una enfermera colombiana explicó que no tenía tiempo para los pacientes (3).

Eficiencia. A diferencia de lo ocurrido en Chile, el gasto para la salud en Colombia ha aumentado mucho: el aporte del Gobierno pasó del 1,39\% del producto interno bruto en 1988 a 4\% en el año 2000; además, la cuota patronal pasó de $8 \%$ a $12 \%$ del salario, con lo que también aumentó sustancialmente el gasto privado. Se calcula que entre 1984 y 1997, el gasto para la salud aumentó en $178 \%$ y el gasto por persona pasó de US\$ 50,00 a finales de la década de 1980 a US\$ 90,00 a finales de la de 1990 (25). Este aumento tan notable del gasto no ha ido acompañado de un mayor acceso a los servicios ni de una mejor calidad, lo que indica que no ha mejorado la eficiencia. Hay que tener en cuenta que muchas de las personas pobres que ahora están cubiertas por un seguro recibían atención médica en los servicios públicos de salud antes de la reforma y que, como ya se ha explicado, muchos pobres asegurados no pueden acceder a los servicios por falta de recursos para pagar la parte que les corresponde de los pagos compartidos, entre otras causas. Además, la adjudicación de los subsidios no se ha hecho adecuadamente; mientras que $30 \%$ de la población que debería recibir el subsidio no lo recibe, $31 \%$ de las personas que reciben subsidios no son pobres (26).

\section{Descentralización: de la teoría a la práctica}

Las agencias internacionales han ofrecido argumentos difíciles de refutar a primera vista para justificar la descentralización de los servicios de salud. Según ellas, cuando personas ajenas a la comunidad, que desconocen la realidad de los usuarios, toman las decisiones y la gerencia de los servicios está bajo la responsabilidad de burócratas con poco contacto con la comunidad, se incrementa la posibilidad de que los servicios no respondan a las necesidades locales y de que su administración sea poco eficiente. Sus promotores argumentan que al descentralizarse los servicios, los usuarios se encuentran más próximos a los que toman las decisiones y pueden asegurarse de que no haya desviaciones entre la política y su aplicación y controlar mejor el uso adecuado de los recursos. La cercanía a los encargados de tomar las decisiones también incrementa las posibilidades de que la comunidad influya en ellas y por lo tanto, se convierte en un mecanismo de promoción de la democracia. Si hay 
negligencia, abusos o corrupción, es más fácil para los usuarios hacer las reclamaciones oportunas. En definitiva, el control y la participación ciudadana aumentan la eficiencia, la calidad y la satisfacción de los usuarios. Sin embargo, un examen basado en datos científicos revela que estos argumentos no reflejan toda la realidad.

El concepto de descentralización es demasiado indefinido para ser útil. Dentro de las funciones públicas sanitarias, el cuadro de toma de decisiones es muy amplio. Cualquier política de promoción de la descentralización debe empezar por identificar las funciones - de las muchas que existen en un sistema de salud-y el nivel (provincial, distrital o municipal) al cual deben y pueden descentralizarse (27). La descentralización de cada función tendrá ventajas e inconvenientes de tipo económico y político que pueden ser diferentes en cada estado o provincia de un país. Las diferencias demográficas, étnicas, epidemiológicas, de desarrollo económico, de infraestructura, de recursos humanos, históricas y políticas entre las grandes ciudades y los municipios pobres en América Latina son tan extremas que no es aconsejable descentralizar las mismas funciones a los mismos niveles en todo el país. Hacerlo sin crear mecanismos que compensen esas diferencias conduce, como se verá más adelante, a resultados indeseables.

Los promotores de la descentralización en América Latina no han estudiado los costos y beneficios políticos, económicos y sociales de cada una de las funciones que se pueden descentralizar. La descentralización, con raras excepciones, ha sido una política improvisada (17), impuesta por el Banco Mundial con el apoyo de otras agencias multilaterales y bilaterales. En los pocos países en que se han aplicado mecanismos compensatorios para eliminar la brecha entre regiones de diferentes niveles de desarrollo, esas medidas no han dado resultado, ya que la descentralización ha aumentado la inequidad (4).

Los estudios antropológicos y sociológicos indican que las comunidades -entre ellas las más pobres- están estratificadas y raramente las elites locales utilizan su poder para resolver los problemas de su comunidad. Según algunos informes y evaluaciones, los políticos no tienen inconvenientes en desviar hacia otros programas recursos asignados para la salud o adjudicarlos dentro del sector sanitario a programas que no son prioritarios, si a su juicio con ello ganan votos y popularidad (28). En muchas comunidades, los encargados de tomar decisiones locales carecen de conocimientos técni$\cos$ que les permitan escoger las intervenciones sanitarias más adecuadas (29). Asimismo, las prioridades expresadas por una comunidad con frecuencia pugnan con principios sanitarios bien esta- blecidos (30), ya que pueden privilegiar la atención médica antes que la prevención, la aplicación de las tecnologías más modernas antes que la atención primaria o tratamientos con medicamentos antes que intervenciones educativas para cambiar el estilo de vida.

No se puede pasar por alto que muchos de los problemas de salud que enfrentan las comunidades se han creado fuera de ellas. Tal es el caso de la desnutrición, los accidentes laborales y las intoxicaciones, así como de otros problemas de salud producidos por condiciones ambientales de las cuales la comunidad tiene poco control o un control muy limitado. Para las empresas transnacionales es más fácil eludir la regulación y los controles locales que los nacionales. Por lo tanto, no es correcto afirmar que las autoridades locales, por conocer las necesidades y demandas de la población, pueden adecuar mejor el uso de los recursos a la realidad local y mejorar la eficiencia y la calidad de los servicios.

Quizá por estas razones no es posible encontrar en la literatura independiente sobre las reformas neoliberales - es decir, la que no está financiada por el Banco Mundial ni por las otras agencias que se adhieren de forma explícita a sus principios- información que confirme que la descentralización en América Latina ha contribuido a lograr los objetivos que sus promotores habían anticipado. Mas bién, se encuentran estudios llevados a cabo por investigadores independientes que afirman lo contrario $(31,32)$. También hay información que permite afirmar que el gasto incurrido por los gobiernos al poner en marcha la descentralización de forma incompleta y fallida ha sido cuantioso (4).

\section{Una hipótesis para explicar el fracaso}

Investigadores independientes que han estudiado la descentralización han señalado desde hace tiempo que el Banco Mundial y otras agencias internacionales promovían la descentralización por otros motivos. Los objetivos oficialmente declarados solo tenían una función promotora. Según estos investigadores, detrás de la descentralización había dos objetivos básicos: el primero era reducir los gastos del Gobierno central mediante la transferencia de los gastos de salud a las provincias, estados o municipios con el fin de liberar fondos para pagar la deuda externa o sus intereses: es decir, aplicar los ajustes estructurales. El mismo Banco Mundial ha reconocido que este ha sido uno de los objetivos de la descentralización (33). El segundo objetivo, que no ha sido declarado abiertamente, es facilitar la privatización de los servicios. Por ejemplo, la conversión de hospitales públicos en autónomos - con ánimo de lucro o sin él- consigue que funcionen como enti- 
dades independientes que pueden contratar al sector privado para servicios de apoyo, tales como análisis de laboratorio, de diagnóstico o de hostelería. Los municipios pequeños, que no tienen capacidad para organizar sus propios servicios de salud, contratan organizaciones no gubernamentales y esta es una forma de privatizar los servicios y transferir la responsabilidad del Estado al sector privado. La experiencia de México respalda estos argumentos.

\section{VEINTE AÑOS DE DESCENTRALIZACIÓN EN MÉXICO}

México, por su tamaño geográfico y poblacional, por su constitución federativa y por su excesivo centralismo político y económico en el Distrito Federal, reúne las condiciones ideales para justificar la descentralización de los servicios de salud. Los estudiosos de la descentralización mexicana se refieren a dos etapas: la primera transcurrió de 1983 a 1988, y la segunda de 1994 hasta el presente. Este proceso se interrumpió de hecho, aunque no oficialmente, durante el sexenio presidencial de Salinas de Gortari (1988-1994) (34).

Todos los programas sexenales de salud han proclamado su apoyo tanto a la descentralización estatal como a la municipal. La descentralización municipal no llegó a iniciarse y según todos los indicios no se aplicará en mucho tiempo. Se debe aclarar que se trata de descentralizar solo los servicios que provee la Secretaría de Salud (SSA), dirigidos a satisfacer las necesidades de salud de las personas que no cuenta con la cobertura de la seguridad social o de seguros privados (45\% de la población), es decir, de los pobres e indigentes. A este grupo se le conoce en México por población abierta.

\section{La primera descentralización}

En la primera etapa, que coincidió con la grave recesión económica de principios de la década de 1980, solamente 14 de los 31 estados respondieron a la llamada del Gobierno federal y aplicaron el programa de descentralización. El resto de los estados y el Distrito Federal no se sumaron a este proceso, pues intuyeron, como sucedió, que se trataba de descentralizar solamente los problemas y no los recursos. El Gobierno federal pidió a los estados descentralizados que aumentaran su contribución al sector de la salud. La dificultad de los estados para contribuir al presupuesto de salud, como al de otros sectores, se debe a que a lo largo de los años su potestad de cobrar impuestos ha ido pasando al Gobierno federal y esto no se revirtió con la descentralización.
El saldo de la primera etapa fue muy negativo. El Gobierno federal no transfirió la autoridad para tomar decisiones en las tres áreas fundamentales de gerencia: programática, de recursos humanos y financiera. Los programas verticales - que abarcan un buen número de programas de salud-continuaron sin ningún cambio, mientras se seguían poniendo en marcha los demás programas de acuerdo con los protocolos de la SSA, aun cuando las características epidemiológicas locales indicasen la necesidad de aplicar variaciones. La posibilidad de que los estados establecieran nuevos programas estaba limitada por la falta de recursos. En el área de recursos humanos, los nombramientos, el destino y la composición de la fuerza laboral seguían centralizados en la SSA. Asimismo, el control de los recursos financieros siguió centralizado en el Gobierno federal y la dependencia financiara de los estados descentralizados del Gobierno federal incluso aumentó (35). Los efectos negativos más graves de esta etapa de la descentralización fueron el incremento de la inequidad y la reducción de la calidad de los servicios. Por ejemplo, un programa patrocinado por el Instituto Mexicano de Seguridad Social (IMSS) y la Coordinación General del Plan Nacional de Zonas Deprimidas y Grupos Marginales (Coplamar) ofrecía servicios hospitalarios y de atención primaria en zonas rurales pobres. Este programa, que estaba dando excelentes resultados desde hacía varios años, fue descentralizado a las secretarías de salud estatales sin transferirle los fondos necesarios para mantener la calidad de los servicios $(36,37)$. El costo de la primera etapa de la descentralización en los 14 estados fue de unos US\$ 452 millones, según cálculos oficiales (38).

\section{La segunda descentralización}

El comienzo de la segunda etapa de la descentralización en 1994 coincidió también con el inicio de una grave crisis financiera y con el establecimiento de un nuevo Gobierno. El proceso fue más lento debido a la experiencia negativa de la primera etapa y a la renuencia de algunos estados a aceptar la descentralización. Esta reticencia solo pudo superarse en algunos estados gracias a presiones políticas. Los Acuerdos de Descentralización, que determinaban las responsabilidades de los estados y de la federación, empezaron a firmarse en 1997 y terminaron con la firma del estado de Chihuahua en 1999 (39).

La transferencia del poder programático ha sido mínima y los programas verticales han seguido sin cambios $^{3}$. Los estados debían pedir autorización

\footnotetext{
3 Homedes N, Ugalde A. Reforming the state: the decentralization of health services in México. 2005. (Manuscrito inédito. Disponible a quién lo solicite).
} 
para establecer programas nuevos con fondos propios y la SSA debía aprobar su forma de funcionamiento. En esta etapa se terminó la homologación de todos los empleados estatales a federales, lo que representó un gasto adicional de aproximadamente US\$ 35 millones anuales, según cálculos realizados en el año 2000 (39). Las decisiones sobre la contratación de personal seguían controladas por la SSA, aunque se autorizó a los estados a transferir trabajadores de un lugar de trabajo a otro. Lo más importante fue la flexibilidad en el uso de fondos federales a partir de la creación del Fondo de Aportaciones para los Servicios de Salud (FASSA) en 1998. Desde entonces, los servicios estatales de salud pueden disponer libremente de los recursos transferidos por el Gobierno federal, excepto de los fondos destinados al salario del personal, que constituye la mayor parte.

Estudios realizados en diferentes estados han documentado que durante la segunda etapa aumentó la inequidad dentro de los propios estados ${ }^{3}$. Según información oficial, también se incrementó la inequidad entre los estados. Así, el estado que tiene la asignación de fondos más alta para la salud tiene un gasto per cápita 156 veces mayor que el que tiene la menor asignación (40). La descentralización no ha contribuido a corregir esa brecha, sino que al contrario, la ha aumentado y los estados más ricos reciben más fondos de las cuotas de recuperación que los estados más pobres. Los fondos federales asignados como aporte de solidaridad para disminuir las inequidades entre los estados han sido tan escasos que no se ha notado su impacto.

\section{La nueva reforma}

En 2003, el Congreso Federal aprobó por gran mayoría la reforma de la Ley de Salud General. Una de las modificaciones de la Ley fue la creación de un seguro popular para la población abierta. El seguro popular surgió en 2004 como un programa vertical al que los estados se podían adscribir libremente y en la actualidad participan todos, excepto el Distrito Federal. Una vez adscritos al seguro popular, los estados tienen que seguir las normas definidas por la SSA, y las familias inscritas -excepto aquellas cuyos ingresos se encuentran en los dos deciles más bajos- pagan una póliza a la SSA y quedan exentas de pagar cuotas de recuperación cuando reciben los servicios. Al dejar de percibir las cuotas, las secretarías estatales pierden la fuente más importante de ingresos, de la cual tenían antes un control completo (41). Sin estos fondos, el concepto de descentralización queda muy desvirtuado. Si se tiene en cuenta que la atención médica constituye el núcleo básico de las actividades de las secretarías estatales y que varios de los programas de salud pública siguen siendo verticales, se entiende que la aplicación del seguro popular lleve, de hecho, a una centralización del sector de la salud.

Para la SSA, la descentralización se ha convertido en un inconveniente que le impide llevar a cabo las reformas que el equipo actual de salud considera necesarias para reducir las inequidades en el sector. Según Frenk y colaboradores, el proceso de descentralización continúa creando barreras al fortalecimiento del desarrollo institucional de los estados e incluso ha exacerbado la desigualdad financiera entre los estados, tema que se está estudiando en las reformas de 2003 (42).

Esta misma idea la había expuesto el Banco Mundial, que finalmente parecía haber aceptado las consecuencias negativas de la descentralización cuando no existen, como en el caso de México, las condiciones necesarias. Según el Banco Mundial, la descentralización puede haber contribuido en gran medida a la fragmentación de las políticas de salud en México, al aumento de las desigualdades y a la reducción de la responsabilidad de los estados de usar eficientemente los recursos federales destinados a los servicios de los pobres (43).

Según el equipo actual que dirige la política de salud de México, se promueve que los estados contraten en el sector privado servicios médicos cubiertos por el seguro popular y que los beneficiarios de ese seguro puedan acceder a los proveedores de salud que deseen, ya sean del sector privado, del público o de la seguridad social. Es decir, se está promoviendo el pluralismo estructurado (5) o la competencia regulada de los servicios de salud.

\section{DISCUSIÓN}

Las reformas de salud neoliberales en América Latina no han tenido el impacto esperado. La historia de descentralización en México, que ya cuenta con veinte años, confirma que este proceso, promovido por las reformas neoliberales, tiene por objetivo reducir las aportaciones de los Gobiernos centrales a los servicios sociales, entre ellos los de salud, para liberar fondos durante las crisis económicas. En México, por ejemplo, las dos etapas de descentralización coincidieron con graves recesiones económicas. También corrobora que la descentralización neoliberal no consiguió los objetivos que anunciaban sus promotores, tales como mejorar la calidad, la eficiencia, la equidad y la participación comunitaria. Por el contrario, en los países latinoamericanos, las reformas han contribuido a aumentar la desigualdad y, posiblemente, a usar los recursos menos eficientemente. El caso de México enseña, además, que la descentralización puede ser sumamente cos- 
tosa. Por lo tanto, es posible responsabilizar a los promotores de la descentralización neoliberal por el daño económico causado a México y a otros países.

Se debe destacar que cuando el modelo de descentralización aplicado en México resultó siendo un obstáculo para el proceso de privatización, se declaró que la descentralización no era tan necesaria como se había pensado y se descartó. Este hecho indica claramente que el objetivo más importante de las reformas liberales es la privatización de los servicios.

Tanto en Chile como en Colombia, la privatización ha perpetuado la inequidad en el acceso a los servicios de salud y no hay pruebas de que haya contribuido a mejorar la calidad ni la eficiencia de los sistemas. Sin embargo, la privatización ha contribuido a que aumenten considerablemente los gastos administrativos y al enriquecimiento de los ISAPRE en Chile y de las administradoras de salud (EPS y ARS) en Colombia. Los que defienden el modelo neoliberal afirman que es demasiado pronto para evaluar el impacto de la reforma y que con mejores sistemas de información y más regulación se pueden obtener los resultados esperados. Sin embargo, los países latinoamericanos no están en condiciones de poder regular el mercado de salud de forma eficaz.

La provisión privada de servicios públicos (agua, electricidad, carreteras, teléfonos, etc.) no es novedosa y en muchos países algunos de estos servicios están privatizados y funcionan bien, gracias a que el Estado ejerce una rigurosa acción reguladora, lo que no ocurre en la mayoría de los países de la Región (44). Además, en muchos países el Estado administra adecuadamente los servicios públicos. Sin embargo, los servicios de salud tienen sus particularidades y su regulación es mucho más compleja. $\mathrm{Ni}$ aun en los Estados Unidos de América -donde el sistema jurídico está más desarrollado y el cumplimiento de las leyes es más estricto que en los países en vías de desarrollo- se ha logrado regular el comportamiento de las compañías aseguradoras y de los prestadores de salud.

El modelo que promueve la reforma neoliberal tiene muchos elementos en común con el sistema estadounidense, aunque cada vez hay más estudios que identifican los graves problemas del sistema de salud de ese país $(45,46)$ a pesar de su elevado costo. Aunque el gasto per cápita para la salud en los Estados Unidos es de US\$ 6000 , aproximadamente 45 millones de personas no tienen ningún tipo de seguro en ese país y otros 36 millones con seguros limitados o con seguros públicos para pobres no tienen acceso a los servicios de salud cuando los necesitan (47). Incluso, las personas de más de 65 años prefieren el plan del Gobierno (Medicare) que los servicios de un seguro privado (48) y los niveles de cobertura de la vacunación son inferiores a los de muchos países latinoamericanos. A diario, la prensa estadounidense denuncia abusos y corrupción de diferentes empresas del sector de la salud (49). Todo esto indica que se trata de un sistema muy poco equitativo.

La debilidad de las agencias reguladoras en la Región y el fracaso del sistema estadounidense deberían haber alertado a los promotores de las reformas neoliberales de las dificultades de poner en marcha sistemas de salud basados en el mercado privado. El presente análisis corrobora las conclusiones de otros investigadores en el sentido de que las reformas del sector de la salud en América Latina no se basan en datos probatorios y benefician a las aseguradoras $(50,51)$.

\section{CONCLUSIÓN}

Los países de la Región han invertido muchos esfuerzos y recursos en promover una reforma que no responde a los valores ni a la capacidad técnica de las instituciones que la tienen que poner en práctica. Las pruebas acumuladas hasta ahora demuestran que el modelo neoliberal no es capaz de solucionar los problemas del sector de la salud.

No se niega la necesidad de introducir cambios o reformas en los sistemas de salud, pero los cambios deben cimentarse en los principios de solidaridad expresados en Alma Ata y dirigirse a mejorar la eficiencia, la equidad y la calidad de los servicios. América Latina tiene los recursos financieros suficientes y cuenta con muchos ejemplos autóctonos de iniciativas que se hubieran podido reforzar en cada país en lugar de introducir modelos basados en ideologías que no han dado buenos resultados, incluso en los países originarios, y que, como se ha demostrado, tampoco han resuelto los problemas que subsisten en los países latinoamericanos donde se han aplicado. Ha llegado la hora de poner fin a los costosos experimentos neoliberales en el sector de la salud en la Región.

\section{SYNOPSIS}

\section{Neoliberal reforms in health services in Latin America: a critical view from two case studies}

Neoliberal reforms have promoted privatization and decentralization as strategies to improve equity, efficiency, and the quality of health services. In this piece the impact of these reforms in Latin America is critically analyzed, and the impacts of privatization in Colombia and of decentralization in Mexico are detailed. These two cases show that after 10 years of privatization in Colombia and 20 years of decen- 
tralization in Mexico the reforms have had the opposite of the desired effect: They have not improved equity, have increased health expenditures, have not increased efficiency, and have not shown a positive impact on quality. Public health programs in Colombia have deteriorated, while decentralization in Mexico has had a very high cost, without achieving the proposed objectives. It is officially accepted that decentralization in Mexico has increased inequity, and that new reforms implemented in 2003 promote vertical pro- grams. Health systems based on regulated competition are not the most suitable ones for Latin America. Latin American countries should improve their health systems in line with the principles stated in the Declaration of Alma Ata and according to their own national experiences.

Key words: health sector reform, decentralization, privatization, Latin America, Colombia, Mexico.

\section{REFERENCIAS}

1. World Health Organization, United Nations Children's Fund. Alma-Ata 1978: primary health care. Geneva: WHO; 1978.

2. Govindaraj R, Reich MR, Cohen JC. World Bank pharmaceuticals discussion paper. Washington, D.C.: World Bank; 2000.

3. Ugalde A, Homedes N. Las reformas neoliberales del sector de la salud: déficit gerencial y alienación del recurso humano en América Latina. Rev Panam Salud Pública. 2005;17(3):202-9.

4. Homedes N, Ugalde A. Why neoliberal heath reforms have failed in Latin America. Health Policy. 2005;71:83-96.

5. Banco Mundial. Informe sobre el desarrollo mundial 1993. Invertir en salud. Washington, D.C.: Banco Mundial; 1993.

6. Londoño JL, Frenk J. Structured pluralism: towards an innovative model for health system reform in Latin America. Health Policy. 1997;41:1-36.

7. Rodríguez-Monguió R, Infante Campos A. Universal health care for Colombians 10 years after Law 100: challenges and opportunities. Health Policy. 2004;68: $129-42$.

8. Colombia, Ministerio de Protección Social. Lineamientos para el desarrollo del PAB. Santa Fe de Bogotá: Ministerio de Protección Social; 2005. Hallado en: http://www.minproteccionsocial.gov. co/MseContent/newsdetail.asp?id= $7737 \&$ idcompany $=8$. Acceso el 18 de febrero de 2005.

9. Ponencia Proyecto de Ley $052 / 04$. Santa Fe de Bogotá: Senado de Colombia (noviembre 2, 2004).

10. Tono T, Velásquez L, Castañeda L. El impacto de la reforma sobre la salud pública. El caso de la salud sexual y reproductiva. Santa Fe de Bogotá: Fundación Corona, Engender Health Foundation Ford; 2002

11. Ayala Cerna C, Kroeger A. La reforma del sector salud en Colombia y sus efectos en los programas de control de tuberculosis e inmunización. Cad Saude Publica. 2002;18(6):1771-81.

12. Colombia, Alcaldía Mayor de Bogotá. Salud a su hogar. Un modelo de atención primaria en salud para garantizar el derecho a la salud en Bogotá. Santa Fe de Bogotá: Secretaría Distrital de Salud de Bogotá; 2004.
13. Grupo de Economía de la Salud. Cobertura del seguro de salud en Colombia. Observatorio de la Seguridad Social. 2001;1(1):1-11.

14. Céspedes JE, Jaramillo I, Castaño RA. Impacto de la reforma del sistema de seguridad social sobre la equidad en los servicios de salud en Colombia. Cad Saude Publica. 2002;18(4):1003-24.

15. Flórez CE, Tono T. La equidad en el sector salud: una mirada de 10 años. Santa Fe de Bogotá: Fundación Corona; 2002. (Documento de trabajo No. 7).

16. Céspedes JE, Almeida CM, Jaramillo I, Reynales J, Olaya S, Uribe C, et al. Efectos de la reforma de la seguridad social en salud en Colombia sobre la equidad en el acceso y la utilización de los servicios de salud. Rev Salud Pública (Colombia). 2000;2(2):145-64

17. Grupo de Economía de la Salud. Resultados económicos de la reforma a la salud en Colombia. Observatorio de la Seguridad Social. 2003;3(7):1-10.

18. Flórez $\mathrm{CE}$, Tono $\mathrm{T}$. Inequities in health status and use of health services in Colombia: 1990-2000. [Presentación] En: The 2003 Hawaii International Conference on Social Sciences, Honolulu, 12-15 de junio de 2003.

19. Restrepo JH, Arango M, Casas L. Estructura y conducta de la oferta del seguro de salud en Colombia. Observatorio de la Seguridad Social. 2001;1(2):1-10.

20. Colombia, Defensoría del Pueblo. Resultados de la encuesta "Evaluación de los servicios de salud que brindan las empresas promotoras de salud (EPS) -índice de vulneración o cumplimiento de los diferentes componentes del derecho a la salud en el esquema de aseguramiento". Santa Fe de Bogotá: Defensoría del Pueblo; 2003.

21. International Development Research Center. Policy Brief. Effects of social security health reform in Colombia on equity in access and utilization of health services. Ottawa: International Development Research Council; 2002

22. Gómez OL, Carrasquilla G. Factors associated with unjustified Cesarean section in four hospitals in Cali, Colombia. Int J Qual Health Care. 1999;11(5):385-9.

23. Mejía Restrepo S, Vélez Arango AL, Buriticá Arboleda OC, Arango Mejía MC, del Río Gómez JA. La política farmacéu- tica nacional en Colombia y la reforma de la seguridad social: acceso y uso racional de medicamentos. Cad Saude Publica. 2002;18(4):1025-39.

24. McPake B, Yepes FJ, Lake R, Sánchez $\mathrm{LH}$. Is the Colombian health system reform improving the performance of public hospitals in Bogotá? Health Policy Plan. 2003;18(2):182-94.

25. Castaño RA, Arbeláez JJ, Giedion U, Morales LG. Evolución de la equidad en el sistema colombiano de salud. Santiago, Chile: Comisión Económica para América Latina y el Caribe; 2001. (Serie Financiamiento y Desarrollo No. 108)

26. Sarmiento GA, González JI, Rodríguez LA. Eficiencia horizontal y eficiencia vertical del sistema de selección de beneficiarios (SISBEN). Coyuntura Social (Colombia). 1999;21:107-25.

27. Ugalde A, Homedes N. Descentralización del sector salud en América Latina. Gaceta Sanitaria. 2002;16(1):18-29.

28. Ruiz Mier F, Giussani B. Descentralización y financiamiento de la provisión de servicios de salud en Bolivia. Informe de Consultoría a CEPAL, ACDI y ODA. La Paz: Comisión Económica para América Latina y el Caribe; 1996.

29. La Forgia GM, Homedes N. Decentralization of health services in Colombia. A review of progress and problems. A report to the World Bank. Washington, D.C.: World Bank; 1992.

30. Ugalde A. Un acercamiento teórico a la participación comunitaria en la atención de la salud. En: Menéndez EL, ed. Participación social: metodología, problemas y expectativas. El caso de Nicaragua, 1978-1989. México D.F.: Instituto Mora; 1999. Pp. 29-45.

31. Jaramillo I. Evaluación de la descentralización de la salud y la reforma de la seguridad social en Colombia. Gaceta Sanitaria. 2002;16(1):48-53.

32. Bossert T. Decentralization. En: Janovsky K, ed. Health policy and system development. An agenda for research. Geneva: World Health Organization; 1996.

33. Griffin C. Empowering mayors, hospital directors, or patients? The decentralization of health care. En: Burki SJ, Perry G, Dillinger W, eds. Beyond the center. Decentralizing the state. Washington, D.C.: World Bank; 1999. 
34. Flamand G. L. Las perspectivas del nuevo federalismo: el sector salud. Las experiencias en Aguascalientes, Guanajuato y San Luis Potosí. México, D.F.: Centro de Investigaciones y Docencia Económica; 1997. (Documento de trabajo No. 55).

35. Cardozo Brum M. La política de descentralización de servicios de salud. Análisis de su proceso y evaluación de resultados. México, D.F.: Universidad Autónoma Metropolitana (Xochimilco); 1995.

36. González-Block MA, Leyva R, Zapata O, Loewe R, Alagón J. Health services decentralization in Mexico: formulation and results of policy. Health Policy Plan. 1989;4(4):301-15.

37. Gershberg AI. Decentralization and recentralization: lessons from the social science sectors in Mexico and Nicaragua. Washington, D.C.: InterAmerican Development Bank; 1998. (Final Report. RE2/S02).

38. Birn AE. Federalist flirtations: the politics and execution of health services decentralization for the uninsured population in Mexico, 1985-1995. J Public Health Policy. 1999;20(1):81-108.

39. López Bárcena D, Real Mata T. Avances de la descentralización de los servicios de salud en México, 1995-1999. En: de la Fuente JR, López Bárcena, eds. Federalismo y salud en México. Primeros alcances de la reforma de 1995. México, D.F.: Diana; 2001. Pp. 13-34.
40. González PE. Federalismo en salud y reforma financiera en México. En: Foro de Federaciones (Ottawa) e Instituto Nacional para el Federalismo y Desarrollo Municipal, eds. México, D.F.: Instituto Nacional para el Federalismo y Desarrollo Municipal; 2003. Pp. 202-13.

41. Ugalde A. Homedes N. Venda de serviços públicos de saúde no Méxicoum estudo qualitativo. En: Magalhães Bosi ML, Mercado FJ, eds. Pesquisa qualitativa de serviços de saúde. Petrópolis: Editora Vozes; 2004. Pp. 564-98.

42. Frenk J, Knaul F, Gómez-Dantés O, González-Pier E, Hernández-Llamas $\mathrm{H}$, Lezana MA. Fair financing and universal social protection: the structural reform of the Mexican health system. México, D.F.: Secretaría de Salud; 2004. Hallado en: http://64.233.187.104/ search?q=cache:ppSO2aPB2egJ:www. salud.gob.mx/pagina_principal/ archivos/03_pub_finan_i.pdf+Frenk $+\mathrm{J}$, $+\mathrm{Knaul}+\mathrm{F},+\mathrm{G} \% \mathrm{C} 3 \% \mathrm{~B} 3 \mathrm{mez}-\mathrm{Dant} \%$ C3\%A9s+Fair+financing+\&hl=es. Acceso el 20 de febrero de 2005.

43. World Bank. Poverty in Mexico: an assessment of conditions, trends and government strategy. Washington, D.C.: World Bank; 2004.

44. Homedes N, Ugalde A. Privatización de los servicios de salud: las experiencias de Chile y Costa Rica. Gaceta Sanitaria. 2002;16(1):54-62.

45. Schoen C, Osborn R, Trang Huynh P, Doty M, Davis K, Zapert K, et al. Pri- mary care and health system performance: adults' experiences in five countries. Health Affairs [publicación periódica en línea] 2004 octubre 28 [citada 2005 febrero 18]. Hallado en: http: / / content.healthaffairs.org / webexclusives/index.dtl?year $=2004$

46. Herbert B. A second opinion. The New York Times, 2004 junio 28; sección A:15 (col. 1).

47. National Association of Community Health Centers. 36 million Americans lack access to basic health care. State-by state reports show that low-income families, minorities hardest hit [press release]. Bethesda, MD: NACHC; 2004 marzo 23.

48. Davis K, Schoen C, Doty M, Tenney K. Medicare versus private insurance: rhetoric and reality. Health Affairs [publicación periódica en línea] 9 de octubre de 2002 [citada 2005 febrero 18]. Hallado en: http://content.healthaffairs.org/ webexclusives/index.dtl?year $=2002$

49. Court J, Smith F. Making a killing. HMOs and the threats to your health. Monroe: Common Courage Press; 1999.

50. Armada F, Muntaner C, Navarro V. Health and social security reforms in Latin America: the convergence of the World Health Organization, the World Bank, and transnational corporations. Int J Health Serv. 2001;31(4):729-68.

51. Waitzkin H. Report of the WHO Commission on Macroeconomics and Health: a summary and critique. Lancet. 2003; 361:523-26.

\section{Convocatoria 2005 Beca Manuel Velasco-Suárez en Bioética}

La Beca Manuel Velasco-Suárez en Bioética, creada en 2002 en honor de un eminente neurólogo mexicano que destacó por sus contribuciones al campo de la bioética, es uno de los cinco galardones otorgados por el Programa de Premios a la Excelencia en Salud Pública Interamericana de la Fundación Panamericana de la Salud y Educación (PAHEF). Mediante la beca se busca estimular el interés de jóvenes docentes e investigadores en la bioética, disciplina que cuenta entre sus principales áreas de interés la justicia y equidad en la distribución de los recursos destinados a la salud, los derechos de los pacientes y las implicaciones éticas y morales de los adelantos biotecnológicos.

La persona ganadora recibirá una beca de US\$10 000, un diploma de honor y un viaje con los gastos pagados a la ciudad de Washington, D.C., donde pronunciará un discurso ante los ministros de salud de países de la Región, sus respectivas delegaciones e invitados de honor que asistan a la reunión anual del Consejo Directivo de la OPS. La fecha límite para recibir las candidaturas es el 6 de mayo de 2005.

Si desea mayor información, vaya al sitio www.pahef.org. También puede escribir a la dirección electrónica de PAHEF, que es la info@pahef.org, o llamar al teléfono (202)974-3416. 